\title{
Ensemble-Based Molecular Simulation of Chemical Reactions under Vibrational Nonequilibrium
}

\author{
Kristof M. Bal, ${ }^{*}$ Annemie Bogaerts, and Erik C. Neyts \\ Research Group PLASMANT, Department of Chemistry, University of Antwerp \\ Universiteitsplein 1, 2610 Antwerp, Belgium \\ E-mail: kristof.bal@uantwerpen.be
}




\section{Computational details}

\section{Deuterium exchange}

Simulations were carried out using LAMMPS, ${ }^{1}$ patched with PLUMED2. ${ }^{2}$ The interatomic potential was the $\mathrm{BOP} \mathrm{Cu}-\mathrm{H}$ force field ${ }^{3}$ and the time step was $0.1 \mathrm{fs}$.

The background temperature in the free energy simulations was kept at $T_{\mathrm{bg}}=300 \mathrm{~K}$ using a colored noise GLE thermostat, ${ }^{4}$ optimized for efficient canonical sampling of frequencies between 0.4 and $4000 \mathrm{~cm}^{-1}{ }^{5}$ The (apparent) free energy surface $F^{*}(s)$ was obtained in sampling runs of $2.5 \mathrm{~ns}$. The collective variable $s$ was $s=r_{\mathrm{AB}}-r_{\mathrm{BC}}$, describing the reaction $\mathrm{D}_{\mathrm{A}} \cdots \mathrm{D}_{\mathrm{B}}-\mathrm{D}_{\mathrm{C}}$. Harmonic restraints were used to limit the $r_{\mathrm{AB}}$ and $r_{\mathrm{BC}}$ to $2.5 \AA$ and to prevent the interaction between $\mathrm{D}_{\mathrm{A}}$ and $\mathrm{D}_{\mathrm{C}}$. The bias potential $V(s)$ was constructed using VES $^{6}$ in its well-tempered variant, ${ }^{7}$ using a 48 th order expansion of Chebyshev polynomials between -2.5 and $2.5 \AA$, and bias factor $\gamma=30$. The bias was updated in steps of $0.25 \mathrm{kcal} / \mathrm{mol}$ every $5 \mathrm{ps}$.

For the sampling of $p^{*}(u)$, the $\mathrm{D}-\mathrm{D}$ distance in the $\mathrm{D}_{2}$ molecule was used as collective variable $u$, which was sampled at each target $T_{\text {vib }}$ during 100 ps. Because the $\mathrm{D}-\mathrm{D}$ vibration is decoupled from all other modes of the $\mathrm{D}_{2}$ molecule, the same GLE thermostat was used as in the free energy calculations, of which the (global) target temperature was set to the desired $T_{\mathrm{vib}}$.

The bias $V(u)$ was parameterized with VES, using $p^{*}(u)$ as a target distribution, a 48th order expansion of Chebyshev polynomials between 0 and $3 \AA$, updated with an interval of $1 \mathrm{ps}$ in steps of $0.1 \mathrm{kcal} / \mathrm{mol}$ over a time of $250 \mathrm{ps}$. The temperature in the system was kept at $T_{\mathrm{bg}}=300 \mathrm{~K}$, yet again using the optimal sampling GLE thermostat. The resulting $V(u)$ was applied to both $r_{\mathrm{AB}}$ and $r_{\mathrm{BC}}$ in each subsequent free energy simulation. This means that two instances of $V(u)$ were applied simultaneously, ensuring that a vibrationally adiabatic process is modeled; the stretch modes of both $r_{\mathrm{AB}}$ and $r_{\mathrm{BC}}$ are excited whenever these bonds exist. 
Next to the implicit treatment of vibrational excitation, we also calculated reaction rate coefficients explicitly. There, nonequilibrium was imposed using the hot spot variant of the GLE thermostat, ${ }^{8}$ explicitly keeping $T_{\mathrm{bg}}$ at $300 \mathrm{~K}$, while imposing a higher $T_{\mathrm{vib}}$ on the D-D bond by exciting a distribution of frequencies centered around $\omega_{0}=3000 \mathrm{~cm}^{-1}$ with a width $\Delta \omega=500 \mathrm{~cm}^{-1}$. Reaction rate coefficients $k$ were calculated at each $T_{\text {vib }}$ by counting the number of reactive events and dividing them by the sampling time of $10 \mathrm{~ns}$ (or $200 \mathrm{~ns}$ for $\left.T_{\text {vib }}=1000 \mathrm{~K}\right)$.

We note that shorter sampling times could be used for the free energy calculations (i.e., in the implicit simulations): rather than tightly converging the bias potential as a measure of the $\mathrm{FES}, F^{*}(s)$ can also be obtained after reweighing a short sampling run on top of an unconverged bias potential. ${ }^{6}$ The explicit simulations, however, must be sufficiently long to achieve good statistics; at $T_{\mathrm{vib}}=1000 \mathrm{~K}$, only 37 reactive events were recorded after 200 ns. The implicit approach is therefore strongly preferred when doing simulations with more expensive potentials.

\section{Nucleophilic substitution}

Simulations were carried out using $\mathrm{CP} 2 \mathrm{~K},{ }^{9}$ patched with PLUMED2. ${ }^{2}$ Interatomic forces were calculated using the $\mathrm{PM} 6{ }^{10}$ semiempirical quantum chemical Hamiltonian and the time step was 0.5 fs. Hydrogen atoms were substituted by deuterium.

The background temperature in the free energy simulations was kept at $T_{\mathrm{bg}}=300 \mathrm{~K}$ using a colored noise GLE thermostat, ${ }^{4}$ optimized for efficient canonical sampling of frequencies between 0.4 and $4000 \mathrm{~cm}^{-1} .{ }^{5}$ The (apparent) free energy surface $F^{*}(s)$ was obtained in sampling runs of $500 \mathrm{ps}$. The collective variable $s$ was $s=r_{\mathrm{C}-\mathrm{Cl}_{1}}-r_{\mathrm{C}-\mathrm{Cl}_{2}}$, describing the two $\mathrm{Cl}$ atoms exchanging their bond with the central $\mathrm{C}$ atom. Harmonic restraints were used to limit the $\mathrm{C}-\mathrm{Cl}$ distances to $3 \AA$. The bias potential $V(s)$ was constructed using $\mathrm{VES}^{6}$ in its well-tempered variant, ${ }^{7}$ using a 48 th order expansion of Chebyshev polynomials between -2 and $2 \AA$, and bias factor $\gamma=30$. The bias was updated in steps of $0.25 \mathrm{kcal} / \mathrm{mol}$ every 
2 ps.

For the $\mathrm{C}-\mathrm{Cl}$ stretch, the collective variable $u$ was equal to the $\mathrm{C}-\mathrm{Cl}$ distance, sampled between 1 and $3 \AA$. For the $\mathrm{C}-\mathrm{D}$ stretch, $u$ is defined as

$$
u=\frac{1}{3} \sum_{i=1}^{3} \frac{1-\left(\frac{r_{\mathrm{C}-\mathrm{D}_{i}}}{d}\right)^{12}}{1-\left(\frac{r_{\mathrm{C}-\mathrm{D}_{i}}}{d}\right)^{24}}
$$

in which $d=1.1 \AA$. This CV does not explicitly capture a particular vibrational mode, but describes how the average $\mathrm{C}-\mathrm{D}$ distance changes as a function of the temperature. This $\mathrm{CV}$ is defined over the interval $0-1$, over which it was also sampled. For the $\mathrm{C}-\mathrm{Cl}$ stretch, $p^{*}(u)$ was sampled at each target $T_{\text {vib }}$ during $100 \mathrm{ps}$; for the C-D stretch, a shorter time step of 0.25 fs was used for accurate sampling of the fast $\mathrm{C}-\mathrm{D}$ vibration over a shorter interval of 50 ps. Because the $\mathrm{C}-\mathrm{Cl}$ and (lumped) $\mathrm{C}-\mathrm{D}$ modes are fairly decoupled, the same GLE thermostat was used as in the free energy calculations, of which the (global) target temperature was set to the desired $T_{\mathrm{vib}}$.

The bias $V(u)$ was parameterized with VES, using $p^{*}(u)$ as a target distribution, a 48th order expansion of Chebyshev polynomials between 0 and $3.5 \AA(\mathrm{C}-\mathrm{Cl})$ or 0 and $1(\mathrm{C}-$ D), updated with an interval of 1 ps in steps of $0.1 \mathrm{kcal} / \mathrm{mol}$ over a time of $200 \mathrm{ps}$. The temperature in the system was kept at $T_{\mathrm{bg}}=300 \mathrm{~K}$, yet again using the optimal sampling GLE thermostat. For the $\mathrm{C}-\mathrm{Cl}$ stretch, the resulting $V(u)$ was simultaneously applied to both $r_{\mathrm{C}-\mathrm{Cl}_{1}}$ and $r_{\mathrm{C}-\mathrm{Cl}_{2}}$ in each subsequent free energy simulation.

\section{$\mathrm{OH}$ addition on carbon dioxide}

Simulations were carried out using CP2K, ${ }^{9}$ patched with PLUMED2. ${ }^{2}$ Interatomic forces were calculated with the semiempirical self-consistent-charge density-functional tight-binding (SCC-DFTB) approximation, using the standard mio-1-1 parameterization. ${ }^{11}$ Spin polarization was applied in simulations involving the $\mathrm{OH}$ radical. The time step was 0.5 fs. The $\mathrm{OH}$ hydrogen atom was substituted by deuterium. 
The background temperature in the free energy simulations was kept at $T_{\mathrm{bg}}=300 \mathrm{~K}$ using a colored noise GLE thermostat, ${ }^{4}$ optimized for efficient canonical sampling of frequencies between 0.4 and $4000 \mathrm{~cm}^{-1} .{ }^{5}$ The (apparent) free energy surface $F^{*}(s)$ was obtained in sampling runs of $250 \mathrm{ps}$. The collective variable $s$ was the distance $r_{\mathrm{O}-\mathrm{C}}$ between the carbon atom of the $\mathrm{CO}_{2}$ molecule and the $\mathrm{O}$ atom of $\mathrm{OH}$. Harmonic restraints were used to limit $r_{\mathrm{O}-\mathrm{C}}$ to $2.5 \AA$ and maintain the $\mathrm{O}-\mathrm{H}$ bond by keeping it shorter than $1.3 \AA$. The bias potential $V(s)$ was constructed using $\mathrm{VES}^{6}$ in its well-tempered variant, ${ }^{7}$ using a 48 th order expansion of Chebyshev polynomials between 1 and $3 \AA$, and bias factor $\gamma=30$. The bias was updated in steps of $0.5 \mathrm{kcal} / \mathrm{mol}$ every $2 \mathrm{ps}$.

For the $\mathrm{O}-\mathrm{C}-\mathrm{O}$ bend, the collective variable $u$ was equal to the $\mathrm{O}-\mathrm{C}-\mathrm{O}$ angle, sampled between 90 and $180^{\circ}$. Because this mode is fairly decoupled from the stretch modes, the same optimal sampling GLE thermostat was used as in the free energy calculations, of which the (global) target temperature was set to the desired $T_{\text {vib }}$.

For the symmetric stretch, $u$ is defined as

$$
u=\frac{1}{2}\left(\frac{1-\left(\frac{r_{1}}{d}\right)^{12}}{1-\left(\frac{r_{1}}{d}\right)^{24}}+\frac{1-\left(\frac{r_{2}}{d}\right)^{12}}{1-\left(\frac{r_{2}}{d}\right)^{24}}\right),
$$

in which $d=1.15 \AA$ and $r_{1}$ and $r_{2}$ are the first and second $\mathrm{C}-\mathrm{O}$ bond length, respectively. $u$ was sampled between 0 and 1 . During sampling, the mode was selectively excited by the hot spot variant of the GLE thermostat, ${ }^{8}$ explicitly keeping $T_{\mathrm{bg}}$ at $300 \mathrm{~K}$, while imposing a higher $T_{\text {vib }}$ to the stretch mode by exciting a distribution of frequencies centered around $\omega_{0}=1500 \mathrm{~cm}^{-1}$ with a width $\Delta \omega=250 \mathrm{~cm}^{-1}$.

For the asymmetric stretch, $u$ is defined as

$$
u=\frac{1}{2}\left(\frac{1-\left(\frac{r_{1}}{d}\right)^{12}}{1-\left(\frac{r_{1}}{d}\right)^{24}}-\frac{1-\left(\frac{r_{2}}{d}\right)^{12}}{1-\left(\frac{r_{2}}{d}\right)^{24}}\right),
$$

in which $d=1.15 \AA$ and $r_{1}$ and $r_{2}$ are the first and second $\mathrm{C}-\mathrm{O}$ bond length, respectively. 
$u$ was sampled between -0.5 and 0.5 . During sampling, the mode was selectively excited by the hot spot variant of the GLE thermostat, ${ }^{8}$ explicitly keeping $T_{\mathrm{bg}}$ at $300 \mathrm{~K}$, while imposing a higher $T_{\text {vib }}$ to the stretch mode by exciting a distribution of frequencies centered around $\omega_{0}=2500 \mathrm{~cm}^{-1}$ with a width $\Delta \omega=250 \mathrm{~cm}^{-1}$.

$p^{*}(u)$ was sampled at each target $T_{\text {vib }}$ during $100 \mathrm{ps}$, employing a shortened time step of 0.25 fs for improved accuracy. The bias $V(u)$ was then parameterized with VES, using $p^{*}(u)$ as a target distribution, a 48th order expansion of Chebyshev polynomials over the same respective intervals used during sampling, updated with an interval of $1 \mathrm{ps}$ in steps of $0.25 \mathrm{kcal} / \mathrm{mol}$ over a time of $200 \mathrm{ps}$. The temperature in the system was kept at $T_{\mathrm{bg}}=300 \mathrm{~K}$, yet again using the optimal sampling GLE thermostat.

\section{Note about exchanging data between simulations}

Whenever $p^{*}(u)$ or $V(u)$ had to be fed into another calculation - that is, $p^{*}(u)$ needing to be supplied for the parametrization of $V(u)$, or application of $V(u)$ during a free energy calculation - these functions were entered in the form of a grid, using standard grid-based functionalities available in PLUMED.

When interpreting the apparent FES $F^{*}(s)$, we always used the estimation directly based on the VES bias $V(s)$.

\section{Relation between $p^{*}(\boldsymbol{u})$ and the well-tempered distribu- tion}

Sampling in the well-tempered distribution is enhanced because the probability distribution along CVs $\boldsymbol{u}$ is partially flattened according to

$$
p_{\mathrm{WT}}(\boldsymbol{u}) \propto(p(\boldsymbol{u}))^{1 / \gamma}
$$


Essentially, this corresponds to sampling the underlying FES $F(\boldsymbol{u})$ at an elevated temperature $\gamma T_{\mathrm{bg}}$. If one assumes that $F(\boldsymbol{u})$ is independent from the temperature along $\boldsymbol{u}$, this means means that

$$
p_{\mathrm{WT}}(\boldsymbol{u})=p^{*}(\boldsymbol{u}) \quad \text { if } \quad \gamma=\frac{T_{\mathrm{vib}}}{T_{\mathrm{bg}}} .
$$

However, if the entropic contribution to the FES is a function of $\boldsymbol{u}, F(\boldsymbol{u})$ will also change as a function of the actual temperature along $\boldsymbol{u}$, and $p_{\mathrm{WT}}(\boldsymbol{u}) \neq p^{*}(\boldsymbol{u})$.
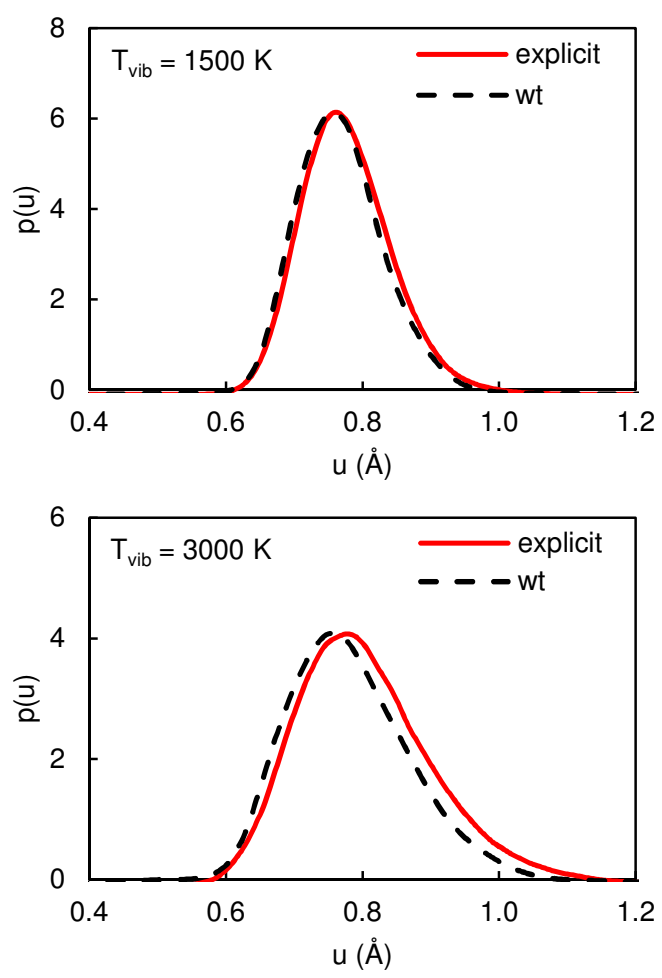

Figure S1: Well-tempered distribution $p_{\mathrm{WT}}(u)$ versus explicit sampling of $p^{*}(u)$ along the D-D bond distance $u$ for two values of $T_{\text {vib }}$, for $T_{\mathrm{bg}}=300 \mathrm{~K}$. Top: $T_{\text {vib }}=1500 \mathrm{~K}$ and $\gamma=5$. Bottom: $T_{\text {vib }}=3000 \mathrm{~K}$ and $\gamma=10$.

This subtle difference is illustrated in Figure S1 for the distribution along the D-D bond for two different values of $T_{\text {vib }}$ (and the corresponding $\gamma$ value when taking $T_{\mathrm{bg}}=300 \mathrm{~K}$ as a reference temperature). $p_{\mathrm{WT}}(u)$ slightly underestimates the tail of $p^{*}(u)$ : the higher entropy of a strongly stretched bond will more strongly contribute to $p^{*}(u)$, which cannot be fully captured by the well-tempered distribution. This difference becomes more pronounced at larger $T_{\mathrm{vib}}$ (or $\gamma$ ). However, when compared to the equilibrium distribution $p(u)$, the 
well-tempered distribution remains very close to $p^{*}(u)$.

\section{Calculating rate coefficients from the Eyring equation}

The Eyring equation gives the relation between the free energy barrier $\Delta^{\ddagger} F^{*}$ and the reaction rate coefficient $k$

$$
k=\frac{1}{\beta h} e^{-\beta \Delta^{\ddagger} F^{*}} .
$$

Essentially, the exponential term is a measure for the probability density in the transition region and serves as an equilibrium constant of sorts; the prefactor is a measure for the flux along the reaction path. What is uncertain in our case, is the definition of $\beta=\left(k_{B} T\right)^{-1}$.

Values of $F^{*}(s)$ were obtained from simulations in an NVT ensemble thermostatted at $T_{\mathrm{bg}}$. Therefore, at least in this simulation, $\beta=\left(k_{B} T_{\mathrm{bg}}\right)^{-1}=\beta_{\mathrm{bg}}$. This means that the probability distribution along the reaction coordinate $s$ under vibrational nonequilibrium is related to the apparent free energy surface according to the relation

$$
F^{*}(s)=-\frac{1}{\beta_{\mathrm{bg}}} \ln p^{*}(s)+C .
$$

Returning to the Eyring equation, we see that its exponential part has to reproduce the probability in the transition region, i.e., recover $p^{*}(s)$. As a result, we must use $T_{\mathrm{bg}}$ in the exponent, because we have made sure that we correctly reproduce $p^{*}(s)$ specifically in simulations that are otherwise at $T_{\mathrm{bg}}$. However, this is not the same temperature that should be used in the prefactor.

The prefactor represents the temperature-dependent frequency of motion along $s$. Especially along this reaction coordinate, vibrationally hot degrees of freedom might play a role. This means that the temperature of $s$ will be between $T_{\mathrm{bg}}$ and $T_{\text {vib }}$. To calculate this temperature $T_{s}$, we must know the relative contribution $\alpha$ of the hot vibration to the overall 
reaction coordinate, which we calculate as follows:

$$
\alpha=1-\frac{\Delta^{\ddagger} F^{*}\left(T_{\mathrm{vib}} \rightarrow \infty\right)}{\Delta^{\ddagger} F^{*}\left(T_{\mathrm{vib}}=T_{\mathrm{bg}}\right)} .
$$

By extrapolating the free energy barrier to the limit of an infinitely hot vibrational mode, we obtain the residual barrier that must be overcome using energy supplied by all remaining degrees of freedom, which are in equilibrium at $T_{\mathrm{bg}}$. This fraction of the barrier is also the fractional contribution of all these "cold" degrees of freedom, and can hence be used to calculate $\alpha$. We then have

$$
T_{s}=(1-\alpha) T_{\mathrm{bg}}+\alpha T_{\mathrm{vib}}
$$

and rewrite the Eyring equation using $\beta_{\mathrm{bg}}$ and $\beta_{s}=\left(k_{B} T_{s}\right)^{-1}$ :

$$
k=\frac{1}{\beta_{s} h} e^{-\beta_{\mathrm{bg}} \Delta^{\ddagger} F^{*}} .
$$

As demonstrated in the main manuscript, this leads to excellent agreement with explicitly computed reaction rate coefficients in the case of the deuterium exchange reaction $(\alpha=0.71)$. Replacing $T$ by $T_{s}$ (and not $T_{\mathrm{bg}}$ ) in the prefactor is the "correction" that is needed to compensate for the indirect - configuration space-based - nature of our method.

Finally, we note that some minor energy leakage can occur while using the hot spot thermostat. This means that the effective $T_{\mathrm{bg}}$ is in some cases slightly higher than its requested value of $300 \mathrm{~K}$. Especially for high-barrier reactions (i.e., low $T_{\text {vib }}$ ) this can result in explicit reaction rates that are too high. In fact, the effective $T_{\mathrm{bg}}$ for $T_{\mathrm{vib}}=1000 \mathrm{~K}$ was found to be $T_{\mathrm{bg} \text {,eff }}=380 \mathrm{~K}$, and the uncorrected explicit rate coefficient was an order of magnitude larger than the implicit one. In that case, we have corrected the rate coefficient by a factor $e^{\left(\beta_{\mathrm{bg}, \mathrm{eff}}-\beta_{\mathrm{bg}}\right) \Delta^{\ddagger} F^{*}}$, which brought the explicit rate coefficient closer to its implicit estimate. The temperature deviations were tiny $(<20 \mathrm{~K})$ in all other cases, and also had a much smaller impact on the effective rate due to the smaller barriers at higher $T_{\text {vib }}$. 


\section{Additional results of the CP2K-based simulations}

Here we report on the profiles of $p^{*}(\boldsymbol{u})$ and $F^{*}(s)$ for the $\mathrm{S}_{\mathrm{N}} 2$ reaction and the $\mathrm{OH}$ addition.

Figure $\mathrm{S} 2$ shows the shape of $p^{*}(u)$ for the two modes of $\mathrm{CD}_{3} \mathrm{Cl}$ considered in the $\mathrm{S}_{\mathrm{N}} 2$ reaction of equilibrium and $T_{\mathrm{vib}}=1500 \mathrm{~K}$, and Figure $\mathrm{S} 3$ does the same for the $\mathrm{CO}_{2}$ modes in the $\mathrm{OH}$ addition reaction.

Figure S4, finally, shows the apparent FESes for both reactions. Here, the equilibrium FES $F(s)$ can be compared to the different apparent FESs $F^{*}(s)$ obtained by exciting each respective mode to $T_{\text {vib }}=1500 \mathrm{~K}$, i.e., by enforcing the various distributions plotted in Figures S2 and S3.
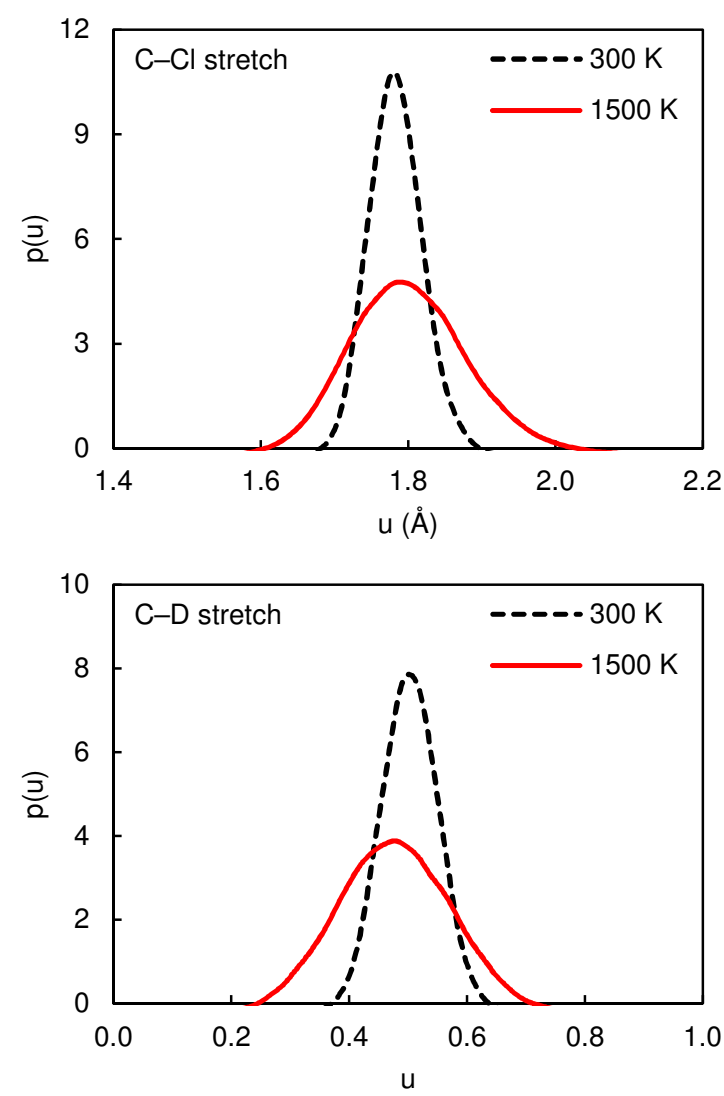

Figure S2: Equilibrium distribution $p(u)$ at $T_{\mathrm{bg}}=300 \mathrm{~K}$ for two considered modes $u$ in $\mathrm{CD}_{3} \mathrm{Cl}$, compared to their respective $p^{*}(u)$ at $T_{\text {vib }}=1500 \mathrm{~K}$. 

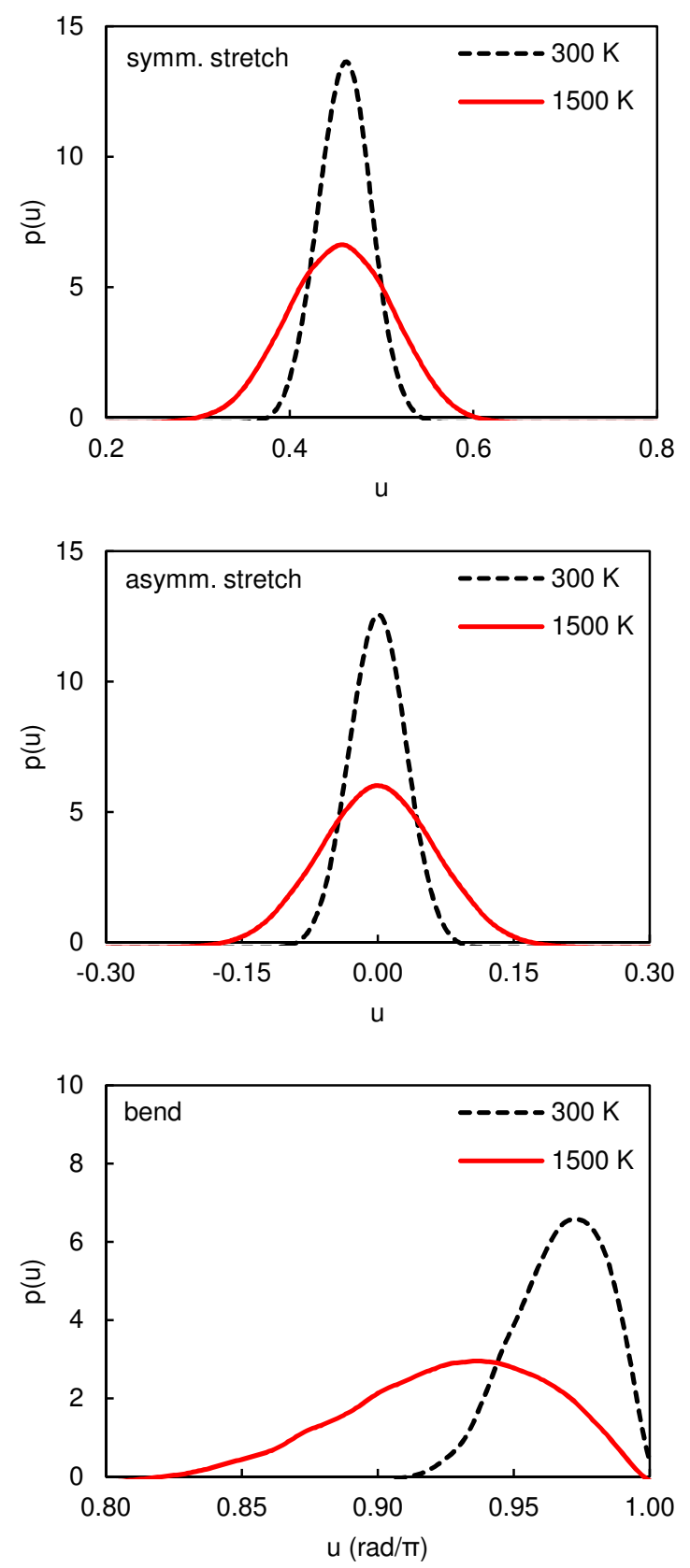

Figure S3: Equilibrium distribution $p(u)$ at $T_{\mathrm{bg}}=300 \mathrm{~K}$ for three considered modes $u$ in $\mathrm{CO}_{2}$, compared to their respective $p^{*}(u)$ at $T_{\mathrm{vib}}=1500 \mathrm{~K}$. 

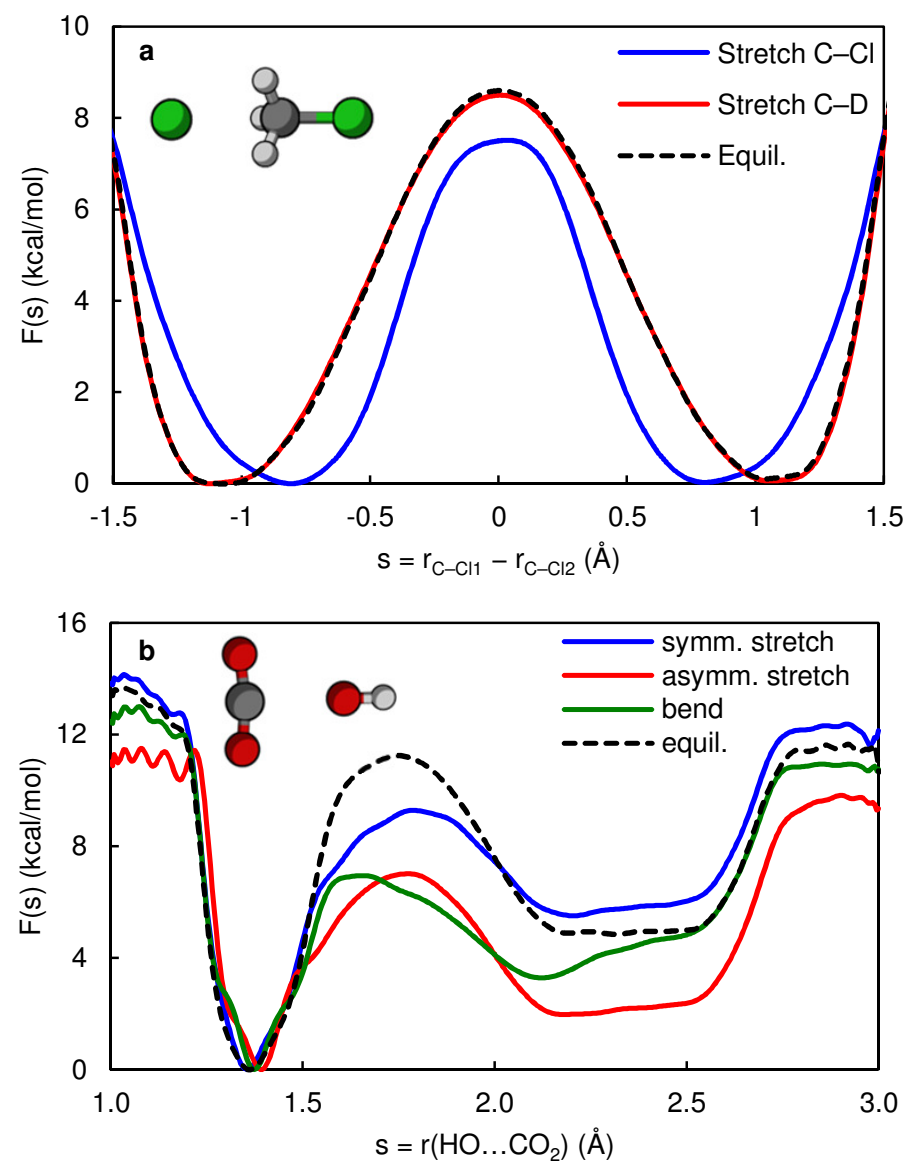

Figure S4: Apparent $F^{*}(s)$ at equilibrium $\left(T_{\mathrm{bg}}=300 \mathrm{~K}\right)$ and conditions with the respective considered modes at $T_{\text {vib }}=1500 \mathrm{~K}$. Top: $\mathrm{S}_{\mathrm{N}} 2$ reaction. Bottom: $\mathrm{OH}$ addition. 


\section{Numerical values of barriers and rates}

\section{Barriers and rates for deuterium exchange}

\begin{tabular}{lccc}
\hline$T_{\text {vib }}(\mathrm{K})$ & $\Delta^{\ddagger} F^{*}(\mathrm{kcal} / \mathrm{mol})$ & $\log k\left(\mathrm{~s}^{-1}\right.$, implicit $)$ & $\log k\left(\mathrm{~s}^{-1}\right.$, explicit $)$ \\
\hline 300 & 11.47 & 4.43 & \\
500 & 10.68 & 5.17 & \\
1000 & 8.34 & 7.13 & 6.99 \\
1500 & 5.55 & 9.34 & 9.45 \\
2000 & 4.70 & 10.07 & 10.11 \\
2500 & 4.20 & 10.52 & 10.51 \\
3000 & 4.04 & 10.72 & 10.71 \\
3500 & 3.94 & 10.85 & 10.99 \\
4000 & 3.88 & 10.95 & 11.14 \\
4500 & 3.85 & 11.03 & 11.27 \\
\hline
\end{tabular}

As noted above the maximal contribution (for $T_{\text {vib }} \rightarrow \infty$ ) of the $\mathrm{D}-\mathrm{D}$ stretch to the overall reaction coordinate is $\alpha \approx 0.71$. 


\section{Barriers for nucleophilic substitution}

\begin{tabular}{lcc}
\hline$T_{\text {vib }}(\mathrm{K})$ & $\Delta^{\ddagger} F_{\mathrm{C}-\mathrm{Cl}}^{*}(\mathrm{kcal} / \mathrm{mol})$ & $\Delta^{\ddagger} F_{\mathrm{C}-\mathrm{D}}^{*}(\mathrm{kcal} / \mathrm{mol})$ \\
\hline 300 & 8.60 & 8.60 \\
500 & 8.36 & 8.51 \\
750 & 8.22 & 8.49 \\
1000 & 7.99 & 8.47 \\
1250 & 7.81 & 8.46 \\
1500 & 7.51 & 8.50 \\
1750 & 7.14 & 8.37 \\
2000 & 6.65 & 8.39 \\
2500 & 5.86 & 8.46 \\
3000 & 4.64 & 8.39 \\
\hline
\end{tabular}

The maximal contribution of $\mathrm{C}-\mathrm{Cl}$ stretch to the overall reaction coordinate is $\alpha_{\mathrm{C}-\mathrm{Cl}} \approx$ 0.60 , and that of the $\mathrm{C}-\mathrm{D}$ stretches is only $\alpha_{\mathrm{C}-\mathrm{D}} \approx 0.02$, of similar magnitudes as the SVP values obtained for substitution on $\mathrm{CH}_{3} \mathrm{I}$ by $\mathrm{F}^{-}$which are 0.78 and 0.03 , respectively. ${ }^{12}$ 


\section{Barriers for $\mathrm{OH}$ addition on carbon dioxide}

\begin{tabular}{lccc}
\hline$T_{\text {vib }}(\mathrm{K})$ & $\Delta^{\ddagger} F_{\text {bend }}^{*}(\mathrm{kcal} / \mathrm{mol})$ & $\Delta^{\ddagger} F_{\text {sym }}^{*}(\mathrm{kcal} / \mathrm{mol})$ & $\Delta^{\ddagger} F_{\text {asym }}^{*}(\mathrm{kcal} / \mathrm{mol})$ \\
\hline 300 & 6.40 & 6.40 & 6.40 \\
500 & 6.14 & 5.43 & 5.79 \\
1000 & 5.12 & 4.37 & 5.39 \\
1500 & 3.66 & 3.78 & 5.04 \\
2000 & 2.46 & 3.38 & 4.83 \\
2500 & 1.50 & 3.06 & 4.60 \\
3000 & 0.83 & 2.83 & 4.42 \\
3500 & 0.77 & 2.61 & 4.41 \\
4000 & 0.67 & 2.38 & 4.29 \\
\hline
\end{tabular}

The maximal contribution of the $\mathrm{O}-\mathrm{C}-\mathrm{O}$ bend to the overall reaction coordinate is $\alpha_{\text {bend }} \approx 0.97$, of the symmetric stretch $\alpha_{\text {sym }} \approx 0.73$ and of the asymmetric stretch is $\alpha_{\text {asym }} \approx 0.40$. 


\section{References}

(1) Plimpton, S. Fast Parallel Algorithms for Short-Range Molecular Dynamics. J. Comput. Phys. 1995, 117, 1-19.

(2) Tribello, G. A.; Bonomi, M.; Branduardi, D.; Camilloni, C.; Bussi, G. PLUMED 2: New Feathers for an Old Bird. Comput. Phys. Commun. 2014, 185, 604-613.

(3) Zhou, X. W.; Ward, D. K.; Foster, M.; Zimmerman, J. A. An Analytical Bond-Order Potential for the Copper-Hydrogen Binary System. J. Mater. Sci. 2015, 50, 2859-2875.

(4) Ceriotti, M.; Bussi, G.; Parrinello, M. Langevin Equation with Colored Noise for Constant-Temperature Molecular Dynamics Simulations. Phys. Rev. Lett. 2009, 102, 020601.

(5) Ceriotti, M.; Bussi, G.; Parrinello, M. Colored-Noise Thermostats à la Carte. J. Chem. Theory Comput. 2010, 6, 1170-1180.

(6) Valsson, O.; Parrinello, M. Variational Approach to Enhanced Sampling and Free Energy Calculations. Phys. Rev. Lett. 2014, 113, 090601.

(7) Valsson, O.; Parrinello, M. Well-Tempered Variational Approach to Enhanced Sampling. J. Chem. Theory Comput. 2015, 11, 1996-2002.

(8) Dettori, R.; Ceriotti, M.; Hunger, J.; Melis, C.; Colombo, L.; Donadio, D. Simulating Energy Relaxation in Pump-Probe Vibrational Spectroscopy of Hydrogen-Bonded Liquids. J. Chem. Theory Comput. 2017, 13, 1284-1292.

(9) Hutter, J.; Iannuzzi, M.; Schiffmann, F.; VandeVondele, J. CP2K: Atomistic Simulations of Condensed Matter Systems. WIREs Comput. Mol. Sci. 2014, 4, 15-25.

(10) Stewart, J. J. P. Optimization of Parameters for Semiempirical Methods V: Modification of NDDO Approximations and Application to 70 Elements. J. Mol. Model. 2007, 13, 1173-1213. 
(11) Elstner, M.; Porezag, D.; Jungnickel, G.; Elsner, J.; Haugk, M.; Frauenheim, T.; Suhai, S.; Seifert, G. Self-Consistent-Charge Density-Functional Tight-Binding Method for Simulations of Complex Materials Properties. Phys. Rev. B 1998, 58, 7260-7268.

(12) Stei, M.; Carrascosa, E.; Dörfler, A.; Meyer, J.; Olasz, B.; Czakó, G.; Li, A.; Guo, H.; Wester, R. Stretching Vibration is a Spectator in Nucleophilic Substitution. Sci. Adv. 2018, 4, eaas9544. 